\title{
Local Fusion Graphs and Sporadic Simple Groups
}

\author{
John Ballantyne \\ School of Mathematics \\ University of Manchester \\ United Kingdom
}

john.ballantyne@manchester.ac.uk

\author{
Peter Rowley \\ School of Mathematics \\ University of Manchester \\ United Kingdom \\ peter.rowley@manchester.ac.uk
}

Submitted: Apr 17, 2014; Accepted: Jul 23, 2015; Published: Jul 31, 2015

Mathematics Subject Classifications: 20D08, 05C25

\begin{abstract}
For a group $G$ with $G$-conjugacy class of involutions $X$, the local fusion graph $\mathcal{F}(G, X)$ has $X$ as its vertex set, with distinct vertices $x$ and $y$ joined by an edge if, and only if, the product $x y$ has odd order. Here we show that, with only three possible exceptions, for all pairs $(G, X)$ with $G$ a sporadic simple group or the automorphism group of a sporadic simple group, $\mathcal{F}(G, X)$ has diameter 2 .
\end{abstract}

Keywords: Local Fusion Graph; Sporadic Simple Group; Diameter

\section{Introduction}

Suppose that $G$ is a finite group with $X$ a $G$-conjugacy class of involutions (that is, a $G$-conjugacy class of elements of order 2). The local fusion graph, $\mathcal{F}(G, X)$, is the graph whose vertex set is $X$ with distinct vertices $x$ and $y$ joined by an edge whenever $x y$ has odd order. Equivalently, $x$ and $y$ are joined if $\langle x, y\rangle$ is a dihedral group of order $2 m, m$ odd, in which case $x$ and $y$ are conjugate in $\langle x, y\rangle$, explaining the graph's epithet. It is clear that $G$ induces graph automorphisms (by conjugation) on $\mathcal{F}(G, X)$ and acts transitively on the vertices. Various properties of local fusion graphs have been investigated in [1] and [2]. In [2] local fusion graphs for finite symmetric groups are studied, the main result being that they always have diameter two, provided that the degree is at least five. The other finite irreducible Coxeter groups are dealt with in [1], which also considers the possible diameters. There, examples are given of groups which have local fusion graphs whose diameter can be arbitrarily large. Local fusion graphs have even being pressed into service [3] in the area of computational algebra, while graphs of a similar nature appear in $[10]$.

Our main result determines the diameter of the local fusion graphs for (most of) the sporadic simple groups and their automorphism groups. We shall follow the notation and 
conventions of the ATLAS [7] and also use it as a source of data on the sporadic simple groups - $\operatorname{Diam}(\mathcal{F}(G, X))$ will denote the diameter of $\mathcal{F}(G, X)$.

Theorem 1. Suppose that $K$ is a sporadic simple group, $G$ a subgroup of $\operatorname{Aut}(K)$ containing $K$, and $X$ is a $G$-conjugacy class of involutions. If $(K, X) \neq(\mathbb{B}, 2 C / 2 D),(\mathbb{M}, 2 B)$ then $\operatorname{Diam}(\mathcal{F}(G, X))=2$.

In Theorem 1 we note that $[G: K]=1$ or 2 (see [7]), so $G=K$ or Aut $(K)$. Before outlining the contents of this paper we introduce some more notation. Suppose that $G$ is a finite group and $X$ is a $G$-conjugacy class of involutions. Let $x, y \in \mathcal{F}(G, X)$ and $i \in \mathbb{N} \cup\{0\}$. We shall use $d(x, y)$ to denote the distance between $x$ and $y$ in $\mathcal{F}(G, X)$, and the $i$-th disc of $\mathcal{F}(G, X), \Xi_{i}(x)$, is defined by

$$
\Xi_{i}(x)=\{y \in X \mid d(x, y)=i\} .
$$

So $\Xi_{0}(x)=\{x\}$, while $\Xi_{1}(x)$ consists of all the neighbours of $x$ in $\mathcal{F}(G, X)$. From now on we fix $t \in X$. For a $G$-conjugacy class $C$ we put

$$
X_{C}=\{x \in X \mid t x \in C\},
$$

and note that $X_{C}$ is invariant under the action of $C_{G}(t)$ by conjugation. We shall sometimes adapt the ATLAS [7] notation for conjugacy classes by adding a subscript which indicates the group whose conjugacy class this is. So, for example, $2 C_{H S: 2}$ indicates that we are considering the $2 C$ conjugacy class (as in the ATLAS) of $H S: 2$.

For most of the sporadic groups, calculations employing GAP [18] and MAGMA [6] yield the diameter of $\mathcal{F}(G, X)$ - the details of these being given in Section 2.1. When $(G, X)=(\mathbb{B}, 2 A)$ or $(\mathbb{M}, 2 A)$, by extracting appropriate subgroup information from the Atlas we demonstrate in Propositions 4 and 6 that $\mathcal{F}(G, X)$ has diameter 2. This approach works largely for the following disparate reasons: the number of $C_{G}(t)$-orbits of $X$ is small; these orbits are of the form $X_{C}$ for some conjugacy class $C$ of $G$; and it is possible to identify $G$-conjugacy classes in smaller subgroups. However, in the case of $(G, X)=(\mathbb{B}, 2 B)$, for example, $X_{2 B}$ is not a $C_{G}(t)$-orbit, so we choose in Proposition 5 to investigate $\mathcal{F}(G, X)$ using the detailed description of the point-line collinearity graph given in [14]. As a by-product, for this case our proof is computer-free. Furthermore, this angle of attack will undoubtedly lead to a sharper picture of the local fusion graph for $(\mathbb{M}, 2 B)$. Indeed, for $(G, X)=(\mathbb{M}, 2 B)$ it can be shown that the diameter of the local fusion graph is at most 6 . This follows from [13], where it is shown that the commuting involution graph of $\mathbb{M}$ on the $2 B$ conjugacy class has diameter 3 , when combined with the observation that two commuting $2 B$ involutions are distance 2 apart in $\mathcal{F}(\mathbb{M}, 2 B)$. However, this bound is almost certainly not the best possible. Finally, we remark that for the three graphs not covered by Theorem 1 the permutation rank of $G$ on $X$ is very large.

We thank the referee for their careful reading of this paper, and their many helpful suggestions. 


\section{Diameter of $\mathcal{F}(G, X)$}

Quite a number of the sporadic simple groups and their conjugacy classes will be dealt with using the next two results. The first is a consequence of some well-known character theoretic results and the second is an elementary observation relating to the size of the first disc of a regular graph. For Lemma 2 we require some more notation, so suppose $G$ is a finite group, with conjugacy classes $\mathcal{K}_{1}, \ldots, \mathcal{K}_{\ell}$ and corresponding class sums $K_{1}, \ldots, K_{\ell}$ in the group algebra $\mathbb{C} G$. Also let $a_{i j k}$ be defined by

$$
K_{i} K_{j}=\sum_{k=1}^{\ell} a_{i j k} K_{k} .
$$

The $a_{i j k}$ are referred to as the structure constants of $G$, and may be calculated from the character table of $G$ (see Chapter 28 of [11] for further details).

Lemma 2. Suppose $G$ is a finite group with $X$ a $G$-conjugacy class of involutions. Assume that $X=\mathcal{K}_{i}$. Then for $x \in X$ we have

$$
\left|\Xi_{1}(x)\right|=\sum_{j} a_{j i i}
$$

where the sum is over all $j$ such that the conjugacy class $\mathcal{K}_{j}$ contains elements of odd order (excluding the conjugacy class of the identity element).

Proof. Let $x \in X$. Then $a_{j i i}$ is the number of pairs $(z, y)$ where $z \in \mathcal{K}_{j}$ and $y \in \mathcal{K}_{i}=X$ are such that $z y=x$. So, letting $\mathcal{K}_{j}$ run over all $G$-conjugacy classes of non-trivial odd order elements, $\sum_{j} a_{j i i}$ is the number of $y \in X$ such that $x y$ has odd order, whence the lemma holds.

Lemma 3. Suppose that $\mathcal{X}$ is a finite regular graph with valency $d$. If $d>|\mathcal{X}| / 2$, then $\mathcal{X}$ is connected and has diameter at most 2.

Proof. For $x \in \mathcal{X}$, let $\Delta(x)$ denote the neighbours of $x$ in $\mathcal{X}$. So $|\Delta(x)|=d$. Let $x \in \mathcal{X}$. Since $|\Delta(x)|=d>|\mathcal{X}| / 2$, the regularity of $\mathcal{X}$ implies connectedness. Suppose there exists $y \in \mathcal{X}$ such that $x$ and $y$ are distance 3 apart. Then $\Delta(x) \cap \Delta(y)=\varnothing$. Therefore

$$
|\Delta(x)| \leqslant|\mathcal{X}|-|\Delta(y)|=|\mathcal{X}|-|\Delta(x)|
$$

by regularity. Hence $|\Delta(x)| \leqslant|\mathcal{X}| / 2$, a contradiction. Thus the diameter of $\mathcal{X}$ is at most 2 .

Note that Lemma 3 is best possible, as the example of dumbbell graphs attest. 


\section{1 $K$ is not isomorphic to $\mathbb{B}$ or $\mathbb{M}$}

In Table 1 we list the first disc sizes for the local fusion graphs of $G$ where $G=K$ or Aut $(K), K$ a sporadic simple group. These values have been calculated using Lemma 2 with the aid of GAP [18]. Note that for

$$
\begin{aligned}
(K, X)= & \left(M_{12}, 2 C\right),\left(J_{1}, 2 A\right),\left(M_{22}, 2 C\right),\left(J_{2}, 2 A\right),\left(J_{3}, 2 A / 2 B\right) \\
& (H e, 2 B),(S u z, 2 A / 2 B),\left(O^{\prime} N, 2 B\right),\left(F i_{22}, 2 A\right),(L y, 2 A) \\
& \left(F i_{23}, 2 A\right),\left(J_{4}, 2 B\right),\left(F i_{24}^{\prime}, 2 C\right)
\end{aligned}
$$

we have $\left|\Xi_{1}(t)\right|>|X| / 2$, and so by Lemma 3 we have $\operatorname{Diam}(\mathcal{F}(G, X))=2$. Now suppose that $(K, X)$ does not fall into this category, and also that $K \neq \mathbb{B}$ or $\mathbb{M}$. Here our strategy is as follows. First we obtain (by means detailed below) a set of $C_{G}(t)$-orbit representatives for $X$. Then for each $C_{G}(t)$-orbit representative $x$ for which $t x$ has even order, we check that there exists another $C_{G}(t)$-orbit representative $y$ for which both ty and $y x$ have odd order, thus demonstrating that $d(t, x)=2$. Then by the vertex-transitivity of $\mathcal{F}(G, X)$ we have $\operatorname{Diam}(\mathcal{F}(G, X))=2$. For a number of cases it is straightforward to obtain a set of $C_{G}(t)$-orbit representatives using the Magma [6] command DoubleCosetRepresentatives to find representatives of the double cosets $C_{G}(t) g C_{G}(t)$, where $g \in G$. However this command can fail when the index $\left[G: C_{G}(t)\right]$ becomes relatively large. Therefore, when $(K, X)=\left(F i_{22}, 2 C\right)$, respectively $\left(F i_{23}, 2 B\right),\left(F i_{23}, 2 C\right),\left(F i_{24}^{\prime}, 2 A\right),\left(F i_{24}^{\prime}, 2 B\right)$ and $\left(F i_{24}^{\prime}, 2 D\right)$, we use the $C_{G}(t)$-orbit representatives calculated on page 119 , respectively pages $128,129,140,82$ and 83 of [17] to verify Theorem 1 , while for $K=C o_{1}$ we make use of the representatives calculated in [4]. Finally, when $K=H N$ and $T h$ the required representatives are taken from [16].

\section{$2.2 \quad K$ is isomorphic to $\mathbb{B}$}

Proposition 4. Suppose that $K \cong \mathbb{B}$ and $X=2 A$. Then $\operatorname{Diam}(\mathcal{F}(G, X))=2$.

Proof. Here we have $G=K \cong \mathbb{B}$. By page 216 of [7], $C_{G}(t)$ has five orbits on $X$, and they are $\{t\}, X_{2 B}, X_{2 C}, X_{3 A}$ and $X_{4 B}$. Clearly $\Xi_{1}(t)=X_{3 A}$. Let $\xi \in 11 A$. Then $C_{G}(\xi)=\langle\xi\rangle \times L$ with $L \cong S_{5}$. Considering elements of order 22 and using [7] we infer that $L \cap 2 A \neq \varnothing \neq L \cap 2 B$. Since there are involutions in $L$ of cycle type $2^{2}$ whose product has cycle type $2^{2}$ and $X_{2 A}=\varnothing$, we must have $L \cap 2 A=(1,2)^{L}$ and $L \cap 2 B=(1,2)(3,4)^{L}$. Without loss we may take $t=(1,2) \in L$ and then for $x=(3,4) \in L$ we have $x \in X_{2 B}$. With $y=(2,3) \in L$ we obtain a path $(t, y, x)$ in $\mathcal{F}(G, X)$. Since $X_{2 B}$ is a $C_{G}(t)$-orbit, this gives $X_{2 B} \subseteq \Xi_{2}(t)$.

Moving on to examine $X_{2 C}$, this time we choose $\xi \in 13 A$. So $C_{G}(\xi)=\langle\xi\rangle \times L$ with $L \cong S_{4}$. Looking at elements of order 26 and employing [7] again we see that $L \cap 2 A \neq$ $\varnothing \neq L \cap 2 C$. Since $X_{2 A}=\varnothing$, we deduce that $L \cap 2 A=(1,2)^{L}$ and $L \cap 2 C=(1,2)(3,4)^{L}$. Again, taking $t=(1,2) \in L$ and $x=(3,4) \in L$ we get $x \in X_{2 C}$ and then arguing as in the case of $X_{2 B}$ gives $X_{2 C} \subseteq \Xi_{2}(t)$.

From [7], looking at elements of order 10 we see $X \cap C_{G}(\xi) \neq \varnothing$ for $\xi \in 5 A$. Now, by [7] $C_{G}(\xi)=\langle\xi\rangle \times L$ where $L \cong H S: 2$. So we may suppose that $t \in L$. Looking 
at products of conjugate involutions in $H S: 2$ we see that $t \in 2 C_{H S: 2}$ and there exists $x \in X_{4 B} \cap L$ (see, for example [5], Table 2). Employing Magma [6], and using the 100 degree permutation representation of $H S: 2$, we check that $d(t, x)=2$ (note that $t$ has 30 fixed points in this permutation representation), which completes the proof.

We shall use $\mathcal{G}$ to denote the point-line collinearity graph of $\Gamma$, the maximal 2-local geometry, for $G \cong \mathbb{B}$. The data arrayed in [14] and [15] which describes the structure of the graph $\mathcal{G}$ will form the backbone of the proof of Proposition 5, and we recommend that the reader has these sources to hand as they are referenced extensively. The vertex set of $\mathcal{G}$ is $X=2 B$. For $x \in X$, the edges of $\mathcal{G}$ joined to $x$ are encoded by the lines in $\Gamma_{x}$, the residue geometry at $x$. Now the lines in $\Gamma_{x}$ correspond to certain type- 2 vectors in the Leech lattice (see [14] and [15] again). We shall display these vectors by writing their co-ordinates on a 24-element set which we denote by $\Omega_{x}$ (the subscript $x$ is to indicate that we are working in $\Gamma_{x}$, as we will be considering a number of different vertices of $\mathcal{G}$ ). Blank entries mean the co-ordinate is zero. Further, the Steiner system $S(24,8,5)$ on $\Omega_{x}$ plays an important role and, just as in [14] and [15], we employ Curtis's MOG (see [9] and also [8]) to describe this Steiner system.

Proposition 5. Suppose that $K \cong \mathbb{B}$ and $X=2 B$. Then $\operatorname{Diam}(\mathcal{F}(G, X))=2$.

Proof. Again we note that $G=K \cong \mathbb{B}$. By Theorem 1(ii) of [14] $G$ has permutation rank 10 on $X$, and the $C_{G}(t)$-orbits are $\{t\}, \Delta_{1}(t), \Delta_{2}^{j}(t)(1 \leqslant j \leqslant 3), \Delta_{3}^{j}(1 \leqslant j \leqslant 4)$ and $\Delta_{4}(t)$. We proceed by examining each of these $C_{G}(t)$-orbits.

$$
\Xi_{1}(t)=\Delta_{3}^{4}(t) \cup \Delta_{4}(t)
$$

From calculation of structure constants and the sizes of the $C_{G}(t)$-orbits (Table 1 of [14]) we see that $\Delta_{3}^{4}(t)=X_{3 A}, \Delta_{4}(t)=X_{5 A}$ and for $x \in X \backslash\left(X_{3 A} \cup X_{5 A} \cup\{t\}\right), t x$ has even order, so giving (5.1).

$$
\Delta_{1}(t) \subseteq \Xi_{2}(t) .
$$

Let $y \in \Delta_{4}(t)$. By Theorem 10 of [14] we may choose $x \in \Delta_{1}(t) \cap \Delta_{3}^{4}(t)$. Then, by (5.1), $(y, t, x)$ is a path in $\mathcal{F}(G, X)$. Since $G$ acts transitively on $X$, there exists $g \in G$ such that $y^{g}=t$. Then $x^{g} \in \Delta_{1}(t)$ with $d\left(t, x^{g}\right)=2$. Because $\Delta_{1}(t)$ is a $C_{G}(t)$-orbit, it follows that $\Delta_{1}(t) \subseteq \Xi_{2}(t)$ and (5.2) holds.

$$
\Delta_{2}^{1}(t) \cup \Delta_{2}^{2}(t) \cup \Delta_{2}^{3}(t) \subseteq \Xi_{2}(t) .
$$

Choose $y \in \Delta_{3}^{4}(t)$. Our aim, for each of $i=1,2,3$, is to find $x_{1}, x_{2} \in \Delta_{1}(y) \cap \Delta_{4}(t)$ so as $x_{2} \in \Delta_{2}^{i}\left(x_{1}\right)$. This would then imply that $\left(x_{1}, t, x_{2}\right)$ is a path in $\mathcal{F}(G, X)$ with $x_{2} \in \Delta_{2}^{i}\left(x_{1}\right)$. The transitively of $G$ on $X$ and the fact that $\Delta_{2}^{i}(t)$ is a $C_{G}(t)$-orbit will then yield (5.3).

Consulting Theorem 9 of [14] we see that choosing $x_{1}, x_{2} \in X$ with $y+x_{j} \in U_{2}(j=$ $1,2)$ will ensure that $x_{1}, x_{2} \in \Delta_{1}(y) \cap \Delta_{4}(t)$. By (4.7) of [15], $\left(\Delta_{2}^{1},+\right) \subseteq U_{2}$. The set $\left(\Delta_{2}^{1},+\right)$ is defined on page 278 of [15], and we recall its definition here. Using the labelling of the MOG [9] on the 24-element set $\Omega$ as in [9], $\left(\Delta_{2}^{1},+\right)$ is the set of all type-2 vectors of 
the Leech lattice whose underlying $\mathcal{C}$-set is an octad contained in $\Omega \backslash\{14, \infty\}$ and which contains 0 . So we fix

$$
y+x_{1}=\begin{array}{|ll|ll|l|}
\hline & & & & \\
2 & 2 & 2 & 2 & \\
\hline 2 & 2 & 2 & 2 & \\
\hline
\end{array}
$$

If we take

$$
y+x_{2}=\begin{array}{|ll|ll|l|}
\hline 2 & 2 & 2 & 2 & \\
\hline 2 & 2 & 2 & 2 & \\
\hline
\end{array}
$$

then $y+x_{2} \in\left(\Delta_{2}^{1},+\right)$ and the inner product $\left(y+x_{1}\right) \cdot\left(y+x_{2}\right)=16$, whence $x_{2} \in \Delta_{2}^{2}\left(x_{1}\right)$ by (3.2)(iii) of [15] and the definition of $\Delta_{2}^{2}\left(x_{1}\right)$. On the other hand, selecting

$$
y+x_{2}=\begin{array}{|ll|l|ll|}
\hline & & & & 2 \\
2 & 2 & & & 2 \\
\hline & & 2 & 2 & 2 \\
& & 2
\end{array} \in\left(\Delta_{2}^{1},+\right)
$$

gives that $\left(y+x_{1}\right) \cdot\left(y+x_{2}\right)=8$. Hence, using (3.2)(iv) of [15] and the definition of $\Delta_{2}^{3}\left(x_{1}\right)$ we have $x_{2} \in \Delta_{2}^{3}\left(x_{1}\right)$.

To complete the proof of (5.3) it remains to find $y+x_{2} \in U_{2}$ such that $x_{2} \in \Delta_{2}^{1}\left(x_{1}\right)$. Now we also have that $\left(\Delta_{2}^{2},-\right) \subseteq U_{2}$ by (4.7) of [15], where $\left(\Delta_{2}^{2},-\right)$ consists of all type-2 vectors in the Leech lattice whose underlying octad of $\Omega$ contains $\{14, \infty\}$ but not 0 . We shall select $y+x_{2}$ from $\left(\Delta_{2}^{2},-\right)$. Taking

$$
y+x_{2}=\begin{array}{|ll|ll|ll|}
\hline & 2 & 2 & 2 & 2 & \\
& & & & & 2 \\
\hline & & & & & 2 \\
& & & & & 2 \\
\hline
\end{array} \quad \in\left(\Delta_{2}^{2},-\right)
$$

we have $\left(y+x_{1}\right) \cdot\left(y+x_{2}\right)=0$. By (3.2) of [15] we now need to show that $\left(y+x_{1}\right)^{\circ} \cap(y+$ $\left.x_{2}\right)^{\circ}=\varnothing$. The sets $\left(y+x_{i}\right)^{\circ}$ are defined prior to (3.2) of [15] - they are certain subsets of $\mathcal{G}^{\circ}$, where $\mathcal{G}^{\circ}$ consists of the following 2-element sets of type- 2 vectors (the numbers in brackets give the number of each type of 2-element set).

$$
\begin{aligned}
& \left\{\left\{4 v_{\infty} \pm 4 v_{j},-4 v_{14} \mp 4 v_{j} \mid j \in \Omega \backslash\{\infty, 14\}\right\}\right. \\
& \left\{\left\{\left(3,-1, \pm 1^{22}\right),\left(1,-3, \pm 1^{22}\right)\right\}\right\} \\
& \left\{\left\{\left(2,-2,( \pm 2)^{6}, 0^{16}\right),\left(2,-2,( \pm 2)^{6}, 0^{16}\right)\right\}\right\}
\end{aligned}
$$

Then

$$
\left(y+x_{i}\right)^{\circ}=\left\{\left\{z_{1}, z_{2}\right\} \in \mathcal{G}^{\circ} \mid\left(y+x_{i}\right) \cdot z_{1}= \pm 16=\left(y+x_{i}\right) \cdot z_{2}\right\} .
$$

First we consider which elements of $\mathcal{G}^{\circ}$ of the form $\left\{4 v_{\infty} \pm 4 v_{j},-4 v_{14} \mp 4 v_{j}\right\}$ are in $\left(y+x_{2}\right)^{\circ}$. Let $Y_{i}$ denote the octad of $\Omega$ underlying $y+x_{i}, i=1,2$. If $j \notin Y_{2}$, then 
$\left(y+x_{2}\right) \cdot\left(4 v_{\infty} \pm 4 v_{j}\right)=8$, so to be in $\left(y+x_{2}\right)^{\circ}$ we must have $j \in Y_{2}$. But for $j \in Y_{2}$, $\left(y+x_{1}\right) \cdot\left(4 v_{\infty} \pm 4 v_{j}\right)=0$ and so $\left\{4 v_{\infty} \pm 4 v_{j},-4 v_{14} \mp 4 v_{j}\right\} \notin\left(y+x_{1}\right)^{\circ}$. Now we look at elements of $\mathcal{G}^{\circ}$ of the form $\left\{\left(2,-2, \pm 2^{6}, 0^{16}\right),\left(2,-2, \pm 2^{6}, 0^{1} 6\right)\right\}$. For such elements to be in $\left(y+x_{2}\right)^{\circ}$ their non-zero co-ordinates must all be located in $Y_{2}$ and hence are not in $\left(y+x_{1}\right)^{\circ}$. Finally, considering the last type of element of $\mathcal{G}^{\circ}$ we see that those in $\left(y+x_{2}\right)^{\circ}$ must look like

$\left\{z_{1}=\begin{array}{|ll|ll|ll}3 & -1 & 1 & 1 & 1 & \\ & & & & 1 \\ \hline( \pm 1)^{16} & & & & 1 \\ & & & & 1\end{array}, z_{2}=\begin{array}{|ll|ll|ll}1 & -3 & -1 & -1 & -1 & \\ & & & & & -1 \\ \hline( \pm 1)^{16} & & & -1 \\ & & & & -1\end{array}\right\}$.

If $\left\{z_{1}, z_{2}\right\} \in\left(y+x_{1}\right)^{\circ}$, then we must have $z_{1}=z_{1}^{\prime}$ or $z_{1}^{\prime \prime}$ where

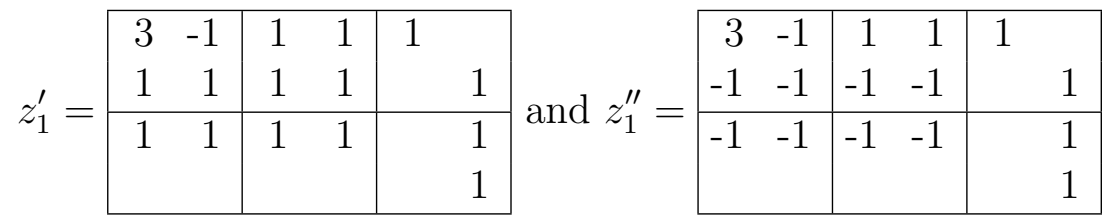

(the blank entries being $\pm 1^{8}$ ). Since $z_{1}$ is required to be a type- 2 vector in the Leech lattice, the 3 and -1 co-ordinates positions of $z_{1}$ must be a $\mathcal{C}$-set of $\Omega$. So if $z_{1}=z_{1}^{\prime}$, then there must be an octad of $\Omega$ containing $\{\infty, 14\}$ and being contained in

$$
\{\infty, 14,15,18,10,2,19,1,12,21\} .
$$

From the MOG we see there are no such octads. Thus $z_{1} \neq z_{1}^{\prime}$ and so $z_{1}=z_{1}^{\prime \prime}$. Then $\Pi=\{\infty, 14,0,8,3,20,4,13,16,17\}$ must be contained in a $\mathcal{C}$-set (which is either a dodecad or a 16 -ad), with

$$
\Omega \backslash \Pi \supseteq \Psi=\{17,11,22,9,5,6\} .
$$

Since the octad containing $\Psi$ is not contained in $\Omega \backslash \Pi$, we must have that $\Pi$ is contained in a dodecad. But this is impossible as $Y_{1} \subseteq \Pi$ and a dodecad cannot contain an octad. Thus we have verified that $\left(y+x_{1}\right)^{\circ} \cap\left(y+x_{2}\right)^{\circ}=\varnothing$ and therefore $x_{2} \in \Delta_{2}^{1}\left(x_{1}\right)$ by $(3.2)$ of [15]. Hence (5.3) is established.

There are three $C_{G}(t)$-orbits remaining which require our attention, namely $\Delta_{3}^{1}(t)$, $\Delta_{3}^{2}(t)$ and $\Delta_{3}^{3}(t)$. In dealing with these we shall first prove the following.

(5.4) Let $x_{1}, x_{2}, x_{3} \in X$ where $x_{2} \in \Delta_{1}\left(x_{1}\right)$ and $x_{3} \in \Delta_{1}\left(x_{2}\right)$. Further assume that $x_{2} \in \Delta_{3}^{4}(t), x_{3} \in \Delta_{4}(t)$ and $x_{3} \in \Delta_{2}^{3}\left(x_{1}\right)$. Then there exists $g \in C_{G}(t)$ and a line $\ell \in \Gamma_{1}\left(x_{3}\right)$ (whose points are $\left.x_{3}, x_{4}, x_{5}\right)$ such that

(i) $x_{4} \in \Delta_{4}(t)$ and $x_{5} \in \Delta_{3}^{2}(t)$; and

(ii) $x_{4}, x_{5} \in \Delta_{3}^{4}\left(x_{1}^{g}\right)$. 
By Theorem 10 of [14] we have that $C_{G}(t) \cap C_{G}\left(x_{2}\right) \cap C_{G}\left(x_{3}\right) \sim M_{22}: 2$ and $x_{3}+x_{2} \in \mathcal{H S}_{x_{3}}$ (the subscript $x_{3}$ telling us that this set of lines are to be viewed in $\Gamma_{x_{3}}$, the residue of $x_{3}$ ). Using the explicit description of $\mathcal{H S}$ given in (3.8) of [15] we may, without loss, assume that $x_{3}+x_{2}=4 v_{\infty}+4 v_{14}$. By hypothesis $x_{3} \in \Delta_{2}^{3}\left(x_{1}\right)$, and so, relative to $x_{1}$, the $C_{G}\left(x_{1}\right) \cap C_{G}\left(x_{3}\right)$ orbits of lines incident with $x_{3}$ are listed in Theorem 5 of [14]. The description of such $C_{G}\left(x_{1}\right) \cap C_{G}\left(x_{3}\right)$ orbits revolves around a certain element of $\Omega_{x_{3}} \backslash\{\infty, 14\}$. Since $C_{G}(t) \cap C_{G}\left(x_{2}\right) \cap C_{G}\left(x_{3}\right)$ acts transitively on $\Omega_{x_{3}} \backslash\{\infty, 14\}$, we may suppose this element is 0 (and replace $x_{1}$ by $x_{1}^{g}$, for some $g \in C_{G}(t) \cap C_{G}\left(x_{2}\right) \cap C_{G}\left(x_{3}\right)$ ). Consulting Theorem 5 of [14] again (applied with $x_{1}^{g}=t$ and $x_{3}=x$ ) we see that the lines in $\left(\alpha_{3}, x_{3}+x_{2}, \pm 3\right)$ have one point in $\Delta_{2}^{3}\left(x_{1}^{g}\right)$ and the other two are in $\Delta_{3}^{4}\left(x_{1}^{g}\right)$. Let $\ell \in \Gamma_{1}\left(x_{3}\right)$ correspond to the following type- 2 vector

$v=$\begin{tabular}{rr|rr|rr|}
\hline 1 & 1 & 1 & 1 & 1 & 1 \\
3 & -1 & -1 & -1 & 1 & 1 \\
\hline-1 & -1 & -1 & -1 & 1 & 1 \\
1 & 1 & 1 & 1 & 1 & 1 \\
\hline
\end{tabular}

and let $x_{3}, x_{4}, x_{5}$ be the three points collinear with $\ell$. Then, as $\ell \in\left(\alpha_{3}, x_{3}+x_{2}, \pm 3\right)$, we have $x_{4}, x_{5} \in \Delta_{3}^{4}\left(x_{1}^{g}\right)$. We now wish to determine the $C_{G}(t)$-orbits to which $x_{4}$ and $x_{5}$ belong. This can be done by pinning down which $C_{G}(t) \cap C_{G}\left(x_{3}\right)$ orbit $\ell$ belongs to and applying Theorem 10 of [14]. Note that $\ell \notin \mathcal{H S}_{x_{3}}$. The inner product of $v$ with the following six type-2 vectors in $\mathcal{H S}_{x_{3}}$
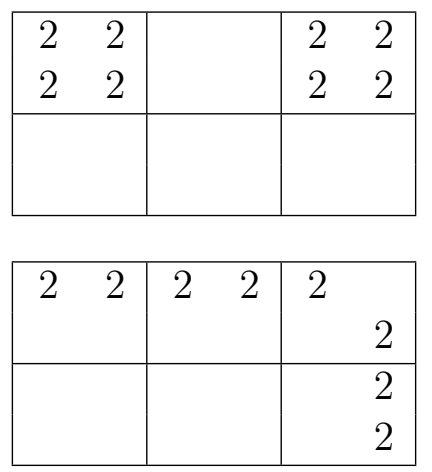

\begin{tabular}{|ll|l|ll|}
\hline 2 & 2 & & 2 & 2 \\
\hline 2 & 2 & & 2 & 2 \\
\hline
\end{tabular}
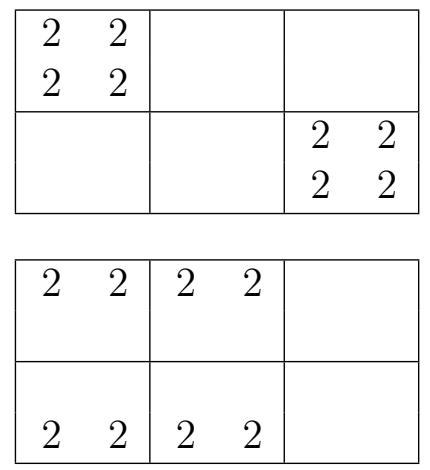

\begin{tabular}{|ll|l|ll|}
\hline 2 & 2 & & & \\
& & & 2 & 2 \\
\hline 2 & 2 & & 2 & 2 \\
\hline
\end{tabular}

is 16. Thus for at least six of the lines $k$ in $\mathcal{H S}_{x_{3}}, \ell \in \alpha_{2}^{2}\left(x_{3}, k\right)$. Surveying the $C_{G}(t) \cap$ $C_{G}\left(x_{3}\right)$-orbits on lines at $x_{3}$ we see the only possibility is that $\ell \in[0,8,28,64]_{\mathcal{H S}_{x_{3}}}$. Hence by Theorem 10 of [14], one of $x_{4}$ and $x_{5}$ is in $\Delta_{3}^{2}(t)$ and the other is in $\Delta_{4}(t)$, which proves $(5.4)$.

$$
\Delta_{3}^{1}(t) \cup \Delta_{3}^{3}(t) \subseteq \Xi_{2}(t)
$$


Choose $x_{2} \in \Delta_{3}^{4}(t)$. Let $x_{1}, x_{3} \in \Delta_{1}\left(x_{2}\right)$ be chosen so as $x_{2}+x_{1}$ corresponds to $4 v_{3}+4 v_{15}$ and $x_{2}+x_{3}$ to the type- 2 vector

$w=$\begin{tabular}{|ll|ll|l|}
\hline & & & & \\
2 & 2 & 2 & 2 & \\
\hline 2 & 2 & 2 & 2 & \\
& & & & \\
\hline
\end{tabular}

Then $x_{2}+x_{1} \in\left(\Delta_{1},-\right) \subseteq U_{3}$ (at $\left.x_{2}\right)$ and $x_{2}+x_{3} \in\left(\Delta_{2}^{1},+\right) \subseteq U_{2}$ (at $\left.x_{2}\right)$ (see (4.7) of [15]), where $\left(\Delta_{1},-\right)$ and $\left(\Delta_{2}^{1},+\right)$ are relative to $0 \in \Omega_{x_{2}}$. Let $\Gamma_{0}\left(x_{2}+x_{1}\right)=\left\{x_{1}, x_{1}^{\prime}, x_{2}\right\}$. Consequently, without loss, we have $x_{1} \in \Delta_{3}^{1}(t), x_{1}^{\prime} \in \Delta_{3}^{3}(t)$ and $x_{3} \in \Delta_{4}^{1}(t)$ by Theorem 9 of [14]. Further the inner product of $4 v_{3}+4 v_{15}$ and $w$ is 8 , whence $x_{3} \in \Delta_{2}^{3}\left(x_{1}\right)$ and $x_{3} \in \Delta_{2}^{3}\left(x_{1}^{\prime}\right)$ by (3.2) of [15]. Applying (2.4.4) to $x_{1}, x_{2}, x_{3}$ yields that there exists $x_{4} \in \Delta_{1}\left(x_{3}\right) \cap \Delta_{4}(t) \cap \Delta_{3}^{4}\left(x_{1}^{g}\right)$ for some $g \in C_{G}(t)$. So, by $(5.1),\left(x_{1}^{g}, x_{4}, t\right)$ is a path of length 2 in $\mathcal{F}(G, X)$. Since $\Delta_{3}^{1}(t)$ is a $C_{G}(t)$-orbit, this forces $\Delta_{3}^{1}(t) \subseteq \Xi_{2}(t)$. A similar argument, with $x_{1}^{\prime}$ in place of $x_{1}$, proves that $\Delta_{3}^{3}(t) \subseteq \Xi_{2}(t)$ also, whence (5.5) follows.

$$
\Delta_{3}^{2}(t) \subseteq \Xi_{2}(t)
$$

We start with $x_{2} \in \Delta_{3}^{4}(t)$, and again let 0 be the element of $\Omega_{x_{2}}$ relative to $t$ which encodes the line orbits at $x_{2}$. Choose $x_{1} \in \Delta_{1}\left(x_{2}\right)$ and $x_{3} \in \Delta_{1}\left(x_{2}\right)$ such that

$$
x_{2}+x_{1}=\begin{array}{|ll|ll|l|}
\hline & 2 & 2 & 2 & \\
\hline 2 & 2 & 2 & 2 & \\
\hline
\end{array}
$$

and

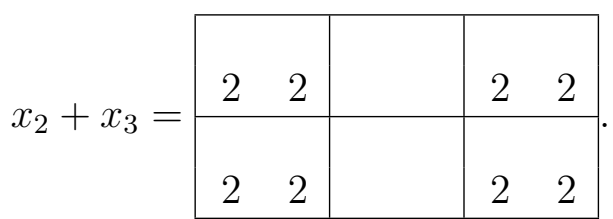

Then both of these type-2 vectors are in $\left(\Delta_{2}^{1},+\right)$ and hence (see (4.7) of [15]) in $U_{2}$ (based at $\left.x_{2}\right)$. Therefore, by Theorem 9 of [14], $x_{1}, x_{3} \in \Delta_{4}(t)$. Moreover, as $\left(x_{2}+x_{1}\right) \cdot\left(x_{2}+x_{3}\right)=8$, $x_{3} \in \Delta_{2}^{3}\left(x_{1}\right)$. So we may apply (2.4.4) to conclude there is an $x_{5} \in \Delta_{1}\left(x_{3}\right)$ with $x_{5} \in \Delta_{3}^{2}(t)$ and $x_{5} \in \Delta_{3}^{4}\left(x_{1}^{g}\right)$ for some $g \in C_{G}(t)$. Hence, as $x_{1}^{g} \in \Delta_{4}(t)$, (5.1) implies that $\left(t, x_{1}^{g}, x_{5}\right)$ is a path in $\mathcal{F}(G, X)$ of length 2. Thus $x_{5} \in \Xi_{2}(t)$, whence $\Delta_{3}^{2}(t) \subseteq \Xi_{2}(t)$, so giving (5.6).

Combining (5.1) - (5.6) completes the proof of Proposition 5.

\section{3 $K$ is isomorphic to $\mathbb{M}$}

Proposition 6. Suppose that $K \cong \mathbb{M}$ and $X=2 A$. Then $\operatorname{Diam}(\mathcal{F}(G, X))=2$.

Proof. Here we have $G=K \cong \mathbb{M}$. By Table 2 of [12], $C_{G}(t)$ has 9 orbits on $X$, namely $\{t\}, X_{2 A}, X_{2 B}, X_{3 A}, X_{3 C}, X_{4 A}, X_{4 B}, X_{5 A}$ and $X_{6 A}$. Hence $\Xi_{1}(t)=X_{3 A} \cup X_{3 C} \cup X_{5 A}$. By 
[7] the eleventh power of any element of $G$ of order 44 is in $4 A$. Also from [7], $G$ has only one conjugacy class of elements of order 11. Let $g$ be an element of $G$ of order 11. Then $C_{G}(g)=\langle g\rangle \times M$ with $M \cong M_{12}$, again by [7]. So $M \cap 4 A \neq \varnothing$. Moreover, looking at elements of order 22 and using [7] once more we deduce that $M \cap 2 A \neq \varnothing \neq M \cap 2 B$. Since elements of order 4 in $M_{12}$ square to the class $2 B_{M_{12}}$ (the $2 B$ class in $M_{12}$ ) and, in $G, 4 A$ elements square into $2 B$, we see that $2 A \cap M=2 A_{M_{12}}$ and $2 B \cap M=2 B_{M_{12}}$. Hence, if $x \in X_{2 A} \cup X_{2 B} \cup X_{4 A}$, we may without loss suppose that $\langle t, x\rangle \leqslant M$ (see, for example, Table 2, line 2 of [5]). Then, by Section 2.1, $d(t, x)=2$.

Now suppose that $x \in X_{4 B}$. Consulting page 234 of [7] we see that $G$ contains a subgroup $H$ where $H \cong A_{6}$, the involutions of $H$ are in $2 A$ and the order four elements of $H$ are in $4 B$. Thus, without loss of generality, $\langle t, x\rangle \leqslant H$, whence $d(t, x)=2$ by Theorem 1.1 of $[2]$.

Finally we assume that $x \in X_{6 A}$. Put $z=t x$ and $H=N_{G}\left(\left\langle z^{2}\right\rangle\right)$. By [7], $z^{2} \in 3 A$ and hence $H \sim 3 \cdot F i_{24}$. Set $\bar{H}=H /\left\langle z^{2}\right\rangle$. In $\bar{H} \backslash \bar{H}^{\prime}$ there are two $\bar{H}$ involution conjugacy classes, namely $2 C_{F i_{24}}$ and $2 D_{F i_{24}}$. Looking at the possible product orders of involutions we see that $\overline{X \cap H}=2 C_{F i_{24}}$ (the Fischer transpositions). So $\bar{t}$ and $\bar{x}$ are transpositions in $F i_{24}$. Thus we may find $y \in X \cap H$ for which $\bar{t} \bar{y}$ and $\overline{y x}$ both have order 3 . Consequently ty and $y x$ have odd order (in fact order 3 ) and so $d(t, x)=2$, whence Proposition 6 holds.

Section 2.1 and Propositions 4, 5 and 6 together prove Theorem 1.

\section{Discs of $\mathcal{F}(G, X)$}

The disc sizes for the local fusion graphs featuring in Theorem 1 are given in Table 1.

Table 1: First disc sizes for $\mathcal{F}(G, X)$

\begin{tabular}{rrrr}
$K$ & Class & Class size & $\left|\Xi_{1}(t)\right|$ \\
\hline$M_{11}$ & $2 \mathrm{~A}$ & 165 & 80 \\
$M_{12}$ & $2 \mathrm{~A}$ & 396 & 180 \\
& $2 \mathrm{~B}$ & 495 & 176 \\
& $2 \mathrm{C}$ & 792 & 460 \\
$J_{1}$ & $2 \mathrm{~A}$ & 1,463 & 1,072 \\
$M_{22}$ & $2 \mathrm{~A}$ & 1,155 & 576 \\
& $2 \mathrm{~B}$ & 330 & 112 \\
& $2 \mathrm{C}$ & 1,386 & 1,040 \\
$J_{2}$ & $2 \mathrm{~A}$ & 315 & 224 \\
& $2 \mathrm{~B}$ & 2,520 & 1,212 \\
& $2 \mathrm{C}$ & 1,800 & 532 \\
$M_{23}$ & $2 \mathrm{~A}$ & 3,795 & 1,344 \\
$H S$ & $2 \mathrm{~A}$ & 5,775 & 2,304 \\
& $2 \mathrm{~B}$ & 15,400 & 7,152 \\
& $2 \mathrm{C}$ & 1,100 & 336
\end{tabular}




\begin{tabular}{|c|c|c|c|}
\hline & $2 \mathrm{D}$ & 23,100 & 10,704 \\
\hline \multirow[t]{2}{*}{$J_{3}$} & $2 \mathrm{~A}$ & 26,163 & 16,832 \\
\hline & $2 \mathrm{~B}$ & 20,520 & 12,716 \\
\hline \multirow[t]{2}{*}{$M_{24}$} & $2 \mathrm{~A}$ & 11,385 & 2,816 \\
\hline & $2 \mathrm{~B}$ & 31,878 & 10,880 \\
\hline \multirow[t]{2}{*}{$M c L$} & $2 \mathrm{~A}$ & 22,275 & 10,304 \\
\hline & $2 \mathrm{~B}$ & 113,400 & 47,564 \\
\hline \multirow[t]{3}{*}{$\mathrm{He}$} & $2 \mathrm{~A}$ & 24,990 & 4, 992 \\
\hline & $2 \mathrm{~B}$ & 187,425 & 119,552 \\
\hline & $2 \mathrm{C}$ & 266,560 & 104,796 \\
\hline \multirow[t]{2}{*}{$R u$} & $2 \mathrm{~A}$ & 593,775 & 149,504 \\
\hline & $2 \mathrm{~B}$ & $1,252,800$ & 570,752 \\
\hline \multirow[t]{4}{*}{ Suz } & $2 \mathrm{~A}$ & 135,135 & 69,632 \\
\hline & $2 \mathrm{~B}$ & $2,779,920$ & $1,454,432$ \\
\hline & $2 \mathrm{C}$ & 370,656 & 137,960 \\
\hline & $2 \mathrm{D}$ & $2,358,720$ & 757,592 \\
\hline \multirow[t]{2}{*}{$O^{\prime} N$} & $2 \mathrm{~A}$ & $2,857,239$ & $1,079,168$ \\
\hline & $2 \mathrm{~B}$ & $2,624,832$ & $1,435,412$ \\
\hline \multirow[t]{2}{*}{$\mathrm{Co}_{3}$} & $2 \mathrm{~A}$ & 170,775 & 59,264 \\
\hline & $2 \mathrm{~B}$ & $2,608,200$ & 904,112 \\
\hline \multirow[t]{3}{*}{$\mathrm{Co}_{2}$} & $2 \mathrm{~A}$ & 56,925 & 14,336 \\
\hline & $2 \mathrm{~B}$ & $1,024,650$ & 379,904 \\
\hline & $2 \mathrm{C}$ & $28,690,200$ & $5,084,672$ \\
\hline \multirow[t]{6}{*}{$F i_{22}$} & $2 \mathrm{~A}$ & 3,510 & 2,816 \\
\hline & $2 \mathrm{~B}$ & $1,216,215$ & 484,352 \\
\hline & $2 \mathrm{C}$ & $36,468,450$ & $12,015,872$ \\
\hline & $2 \mathrm{D}$ & 61,776 & 22,400 \\
\hline & $2 \mathrm{E}$ & $19,459,440$ & $7,102,592$ \\
\hline & $2 \mathrm{~F}$ & $22,239,360$ & $10,969,856$ \\
\hline \multirow[t]{3}{*}{$H N$} & $2 \mathrm{~A}$ & $1,539,000$ & 391,424 \\
\hline & $2 \mathrm{~B}$ & $74,064,375$ & $26,906,624$ \\
\hline & $2 \mathrm{C}$ & $75,240,000$ & $28,083,824$ \\
\hline Ly & $2 \mathrm{~A}$ & $1,296,826,875$ & $659,509,424$ \\
\hline$T h$ & $2 \mathrm{~A}$ & $976,841,775$ & $377,298,944$ \\
\hline \multirow[t]{3}{*}{$F i_{23}$} & $2 \mathrm{~A}$ & 31,671 & 28,160 \\
\hline & $2 \mathrm{~B}$ & $55,582,605$ & $15,234,560$ \\
\hline & $2 \mathrm{C}$ & $12,839,581,755$ & $3,308,650,496$ \\
\hline \multirow[t]{3}{*}{$\mathrm{Co}_{1}$} & $2 \mathrm{~A}$ & $46,621,575$ & $13,451,264$ \\
\hline & $2 \mathrm{~B}$ & $2,065,694,400$ & $902,774,912$ \\
\hline & $2 \mathrm{C}$ & $10,680,579,000$ & $3,014,586,368$ \\
\hline \multirow[t]{2}{*}{$J_{4}$} & $2 \mathrm{~A}$ & $3,980,549,947$ & $1,112,555,520$ \\
\hline & $2 \mathrm{~B}$ & $47,766,599,364$ & $26,545,360,896$ \\
\hline \multirow[t]{4}{*}{$F i_{24}^{\prime}$} & $2 \mathrm{~A}$ & $4,860,485,028$ & $1,504,701,440$ \\
\hline & $2 \mathrm{~B}$ & $7,819,305,288,795$ & $3,351,534,645,248$ \\
\hline & $2 \mathrm{C}$ & 306,936 & 275,264 \\
\hline & $2 \mathrm{D}$ & $5,686,767,482,760$ & $1,780,551,713,600$ \\
\hline
\end{tabular}




$\begin{array}{rrrr}\mathbb{B} & 2 \mathrm{~A} & 13,571,955,000 & 2,370,830,336 \\ & \text { 2B } & 11,707,448,673,375 & 4,010,408,935,424 \\ & \text { 2C } & 156,849,238,149,120,000 & 56,546,114,902,065,152 \\ & \text { 2D } & 355,438,141,723,665,000 & 94,228,887,171,497,984 \\ \mathbb{M} & \text { 2A } & 97,239,461,142,009,186,000 & 30,528,114,911,948,570,624 \\ & \text { 2B } & 5,791,748,068,511,982,636,944,259,375 & 1,486,325,429,210,105,899,724, \\ & & \end{array}$

\section{References}

[1] J. Ballantyne. On Local Fusion Graphs of Finite Coxeter Groups. J. Group Theory, 16(4): 595-617, 2013.

[2] J. Ballantyne, N. Greer, and P. Rowley. Local Fusion Graphs for Symmetric Groups. J. Group Theory, 16(1): 35-49, 2013.

[3] J. Ballantyne, and P. Rowley. A Note on Computing Involution Centralizers. J. Symb. Comp., 54: 1-8, 2013.

[4] C. Bates, and P. Rowley. Involutions in Conway's largest simple group. LMS J. Comput. Math., 7: 337-351, 2004.

[5] C. Bates, D. Bundy, S. Hart, and P. Rowley. Commuting involution graphs for sporadic simple groups. J. Algebra, 316(2): 849-868, 2007.

[6] W. Bosma, J. Cannon, and C. Playoust. The Magma algebra system. I. The user language. J. Symbolic Comput., 24(3-4): 235-265, 1997.

[7] J. H. Conway, R. T. Curtis, S. P. Norton, R. A. Parker, and R. A. Wilson. Atlas of Finite Groups. Maximal subgroups and ordinary characters for simple groups. With computational assistance from J. G. Thackray. Oxford University. Press, Eynsham: 1985.

[8] J. H. Conway, and N. J. A. Sloane. Sphere packings, lattices and groups, Third edition. With additional contributions by E. Bannai, R. E. Borcherds, J. Leech, S. P. Norton, A. M. Odlyzko, R. A. Parker, L. Queen and B. B. Venkov. Grundlehren der Mathematischen Wissenschaften [Fundamental Principles of Mathematical Sciences], 290. Springer-Verlag, New York, lxxiv+703 pp.: 1999.

[9] R. T. Curtis. A new combinatorial approach to $M_{24}$. Math. Proc. Cambridge Philos. Soc., 79(1): 25-42, 1976.

[10] A. Devillers, and M. Giudici. Involution graphs where the product of two adjacent vertices has order three. J. Aust. Math. Soc., 85(3): 305-322, 2008.

[11] G. James, and M. Liebeck. Representations and characters of groups, Second edition. Cambridge University Press, New York: viii+458 pp., 2001. 
[12] S.P. Norton. The uniqueness of the Fischer-Griess Monster. Finite groups - coming of age (Montreal, Que., 1982), 271-285, Contemp. Math., 45, Amer. Math. Soc., Providence, RI: 1985.

[13] P. Rowley. Diameter of the Monster Graph. Unpublished manuscript.

[14] P. Rowley, and L. Walker. A 11,707,448,673,375 vertex graph related to the baby monster. I. J. Combin. Theory Ser. A, 107(2): 181-213, 2004.

[15] P. Rowley, and L. Walker. On point orbits of the $\mathrm{Co}_{2}$-minimal parabolic geometry. J. Combin. Theory Ser. A, 107(2): 263-293, 2004.

[16] P. Rowley, and P. Taylor. Point-line collinearity graphs of two sporadic minimal parabolic geometries. J. Algebra, 331: 304-310, 2011.

[17] P. Taylor. Computational Investigations into Finite Groups. PhD Thesis, University of Manchester: 2011.

[18] The GAP Group. GAP - Groups, Algorithms, and Programming, Version 4.3. http: //www.gap-system.org: 2002. 